\title{
ON THE CAUCHY PROBLEM IN A CLASS OF ENTIRE FUNCTIONS IN SEVERAL VARIABLES
}

\author{
EUGENI LEINARTAS \\ Krasnoyarsk State University \\ Svobodny 79, 660062 Krasnoyarsk, Russia
}

\begin{abstract}
We study the integral representation of solutions to the Cauchy problem for a differential equation with constant coefficients. The Cauchy data and the right-hand of the equation are given by entire functions on a complex hyperplane of $\mathbb{C}^{n+1}$. The Borel transformation of power series and residue theory are used as the main methods of investigation.
\end{abstract}

1. Introduction. For holomorphic partial differential equations the local theory of Cauchy problem is well developed. In the non-characteristic case the classical CauchyKovalevskaya theorem states existence and uniqueness of analytic solutions. Globally, if we have the entire Cauchy data on a hyperplane, the Cauchy-Kovalevskaya theorem can in certain cases be extended to yield entire solutions (cf. results by M.Miyake [1] and J.Persson [2]).

Recently, Sternin and Shatalov have given explicit solutions of the global Cauchy problem in the constant coefficient case with Cauchy data on an arbitrary analytic hypersurface in terms of the Radon-Laplace integral transform (see [3]).

In this paper a new integral representation for the solutions to a class of Cauchy problems is obtained. We assume that Cauchy data and the right-hand of a partial differential equation are entire functions. As an example we consider the case when the characteristic polynomial $P(\tau, \xi)$ is homogeneous polynomial in two variables $(\tau, \xi) \in \mathbb{C}^{2}$.

2. Notation and definitions. We will work in $\mathbb{C}^{n+1}$ using the following variables:

$$
x=\left(x_{1}, \ldots, x_{n}\right) \in \mathbb{C}^{n}, \quad \xi=\left(\xi_{1}, \ldots, \xi_{n}\right) \in \mathbb{C}^{n}, \quad t \in \mathbb{C}, \tau \in \mathbb{C} .
$$

$\mathbb{C}_{x}^{n}$ is considered as a linear subspace of $\mathbb{C}^{n+1}$, i.e. $\mathbb{C}_{x}^{n}=\left\{(t, x) \in \mathbb{C}^{n+1}: t=0\right\}$. $\alpha=\left(\alpha_{1}, \ldots, \alpha_{n}\right) \in \mathbb{N}^{n}$ and $\beta=\left(\beta_{1}, \ldots, \beta_{n}\right)$ are multiindices, $|\alpha|=\alpha_{1}+\ldots+\alpha_{n}$,

1991 Mathematics Subject Classification: 32A25, 32A27.

The paper is in final form and no version of it will be published elsewhere. 
$x^{\alpha}=x_{1}^{\alpha_{1}} \ldots x_{n}^{\alpha_{n}}$. For differential operators we use the following notation:

$$
D_{t}=\frac{\partial}{\partial t}, \quad D_{x}^{\alpha}=\frac{\partial^{|\alpha|} u}{\partial x_{1}^{\alpha_{1}} \ldots \partial x_{n}^{\alpha_{n}}} .
$$

We treat $m$ th order, linear PDEs with constant coefficients of the following type:

$$
D_{t}^{m} u+\sum_{k=0}^{m-1} \sum_{|\alpha| \leq k} a_{k, \alpha} D_{t}^{m-k-1} D_{x}^{\alpha} u=\Phi(t, x)
$$

or for short:

$$
P(D) u=\Phi \text {. }
$$

The corresponding symbol for $P$ is

$$
P(\tau, \xi)=\tau^{m}+\sum_{k=0}^{m-1}\left(\sum_{|\alpha| \leq k} a_{k, \alpha} \xi^{\alpha}\right) \tau^{m-k-1}=\sum_{\mu=0}^{m} b_{\mu}(\xi) \tau^{m-\mu},
$$

where $\tau \in \mathbb{C}, \xi \in \mathbb{C}^{n}, b_{0}(\xi) \equiv 1, b_{\mu}(\xi)=\sum_{|\alpha| \leq \mu} a_{m-\mu, \alpha} \xi^{\alpha}, \mu=1, \ldots, m$. The equation $P(\tau, \xi)=0$ is called characteristic with respect to (1).

We can now formulate the following Cauchy problem. Suppose that $\Phi(t, x)$ is an entire function in $\mathbb{C}^{n+1}$ and that we have entire functions $v_{k}(x)$ in $\mathbb{C}_{x}^{n}$. We seek the unique (entire) solution $u(t, x)$ satisfying

$$
P(D) u=\Phi \quad \text { and }
$$

$$
D_{t}^{k} u=v_{k}(x), \quad k=1, \ldots, m-1, \quad \text { for } t=0 .
$$

Note that since the equation $(1)$ is normal (i.e. $b_{0}(\xi)=1$ ), the Cauchy-Kovalevskaya theorem yields a unique, local solution $u(t, x)$ of $(*),(* *)$. If we find an entire solution $\tilde{u}(t, x)$ of $(*),(* *)$ then $u=\tilde{u}$ by the uniqueness theorem for holomorphic functions.

Definition. Let an entire function $F(x)$ be given by

$$
F(x)=\sum_{|\alpha| \geq 0} \frac{f(\alpha)}{\alpha !} x^{\alpha}
$$

where $\alpha !=\alpha_{1} ! \ldots \alpha_{n} !$ A function $\check{F}(x)$ is called the Borel transform of $F(x)$ if

$$
\check{F}(x)=\sum_{|\alpha| \geq 0} \frac{f(\alpha)}{\xi^{\alpha+I}}, \quad \text { where } \quad I=(1, \ldots, 1) .
$$

We will need the following result ([4]): if $F(x)$ is an entire function of exponential type $\sigma=\left(\sigma_{1}, \ldots, \sigma_{n}\right)$ then $\check{F}(x)$ is holomorphic in $\Gamma_{\sigma}=\left\{x \in \mathbb{C}^{n}:\left|x_{j}\right|>\sigma_{j}>0, j=1, \ldots, n\right\}$. Choose $r_{j}>\sigma_{j}$; then $\check{F}$ is holomorphic in the closed domain $\bar{\Gamma}_{r}=\left\{x \in \mathbb{C}^{n}:\left|x_{j}\right| \geq r_{j}, j=\right.$ $1, \ldots, n\}$.

Let us denote by $\gamma_{\xi}$ the set $\left\{x: \mathbb{C}^{n}:\left|\xi_{j}\right|=r_{j}, j=1, \ldots, n\right\}$. Under the above assumptions we have the integral formula

$$
F(x)=\frac{1}{(2 \pi i)^{n}} \int_{\gamma_{\xi}} \check{F}(\xi) e^{x \xi} d \xi,
$$

where $x \xi=x_{1} \xi_{1}+\ldots+x_{n} \xi_{n}, d \xi=d \xi_{1} \wedge \ldots \wedge d \xi_{n}$. 
3. We can now formulate our main result. We choose $r=\left(r_{1}, \ldots, r_{n}\right)$ so that the functions $\check{v}_{k}(x)$ are holomorphic in $\bar{\Gamma}_{r}$ for $k=0,1, \ldots, m-1$. Then we choose $r_{0}$ satisfying the following conditions:

(i) solutions of $P(\tau, \xi)=0$ belong to $\left\{\tau \in \mathbb{C}:|\tau|<r_{0}\right\}$ for all $\xi \in \gamma_{\xi}$,

(ii) the function $\check{\Phi}(t, x)$ is holomorphic in $\bar{\Gamma}_{r_{0}, r} \subset \mathbb{C}_{t, x}^{n+1}$

Definition. Let $K(\check{v}(\xi), b(\xi), \tau)$ denote the function

$$
K(\check{v}, b, \tau)=\sum_{k=0}^{m-1}\left(\sum_{\mu+\nu=k} \check{v}_{\mu}(\xi) b_{\nu}(\xi)\right) \tau^{m-k-1} .
$$

THEOREM. A solution of the problem $(*),(* *)$ is given by the formula

$$
u(t, x)=\frac{1}{(2 \pi i)^{n+1}} \int_{\gamma_{\tau} \times \gamma_{\xi}} \frac{[K(\check{v}, b, \tau)+\check{\Phi}(\tau, \xi)] e^{t \tau+x \xi} d \tau \wedge d \xi}{P(\tau, \xi)} .
$$

Proof. Substituting (4) into $(*)$ we obtain $P(D) u=I_{1}+I_{2}$, where

$$
\begin{aligned}
& I_{1}=\frac{1}{(2 \pi i)^{n+1}} \int_{\gamma_{\tau} \times \gamma_{\xi}} K(\check{v}, b, \tau) e^{t \tau+x \xi} d \tau \wedge d \xi, \\
& I_{2}=\frac{1}{(2 \pi i)^{n+1}} \int_{\gamma_{\tau} \times \gamma_{\xi}} \check{\Phi}(\tau, \xi) e^{t \tau+x \xi} d \tau \wedge d \xi .
\end{aligned}
$$

Since $K(\check{v}, b, \tau)$ is holomorphic with respect to $\tau$ for all $\xi \in \gamma_{\xi}$ we have $I_{1}=0$. From (2) we conclude $I_{2}=\Phi(t, x)$. It follows that $P(D) u=\Phi(t, x)$. We only need to show that $D^{k} u=v_{k}(x)$ for $t=0, k=0,1, \ldots, m-1$. Substituting (4) in $(*)$ we obtain $\left.D_{t}^{k} u\right|_{t=0}=I_{3}+I_{4}$, where

$$
\begin{aligned}
I_{3} & =\int_{\gamma_{\xi}}\left(\int_{\gamma_{r}} \frac{K(\check{v}, b, \tau) \tau^{k} d \tau}{P(\tau, \xi)}\right) e^{x \xi} d \xi, \\
I_{4} & =\int_{\gamma_{\xi}}\left(\int_{\gamma_{r}} \frac{\check{\Phi}(\tau, \xi) \tau^{k} d \tau}{P(\tau, \xi)}\right) e^{x \xi} d \xi .
\end{aligned}
$$

Expanding $K / P, \check{\Phi} / P$ in powers of $1 / \tau$ we conclude that $\left.D_{t}^{k} u\right|_{t=0}=v_{k}(x)$ (note that we have used (2) again).

4. Example. Let $P(\tau, \xi)$ be a homogeneous polynomial in two complex variables $(\tau, \xi)$. We will denote by $\lambda_{j}$ the roots of $P(\lambda, 1)=0$. For simplicity we assume that $\lambda_{j}$ are simple roots. According to the above remark, we have

$$
\begin{gathered}
P(\tau, \xi)=\prod_{j=1}^{m}\left(\tau-\lambda_{j} \xi\right), \\
b_{k}(\xi)=(-1)^{k}\left(\sum_{1 \leq i_{1}<\ldots<i_{k} \leq m} \lambda_{i_{1}} \ldots \lambda_{i_{k}}\right) \xi^{k}=\sigma_{k}(\lambda) \xi^{k}, \quad k=1, \ldots, m, \\
b_{0}(\xi)=1 .
\end{gathered}
$$


We define $\mathfrak{P}_{\mu}(v)=\mathfrak{P}_{1}\left(\mathfrak{P}_{\mu-1}(v)\right)$ and $\mathfrak{P}_{1}(v)=\int_{0}^{\xi} v(\xi) d \xi$. Under the above assumptions and notations we have

(5) $u(t, x)=\sum_{j=1}^{m} \frac{1}{\left(\prod_{\substack{k=1 \\ k \neq j}}^{m}\left(\lambda_{k}-\lambda_{j}\right)\right)} \sum_{i=0}^{m-1}\left(\sum_{\mu+\nu=i} \mathfrak{P}_{\mu}\left(v_{\mu}\left(x+\lambda_{j} t\right)\right) \sigma_{\nu}(\lambda)\right) \lambda_{j}^{m-i-1}$.

Proof. We first compute (4) by the residue theorem in variable $\tau$, next we use the following generalization of (2):

$$
\int_{\gamma} \xi^{\mu} \check{F}(\xi) e^{x \xi} d \xi=\mathfrak{P}_{\mu}(F(x))
$$

In particular, for the wave equation

we have $m=2, \lambda_{1}=a, \lambda_{2}=-a$; then

$$
\frac{\partial^{2} u}{\partial t^{2}}=a^{2} \frac{\partial^{2} u}{\partial x^{2}}
$$

$$
u(t, x)=\frac{1}{2}\left[v_{0}(x+a t)+v_{0}(x-a t)\right]+\frac{1}{2 a}\left[\mathfrak{P}_{1}\left(v_{1}(x+a t)\right)-\mathfrak{P}_{1}\left(v_{1}(x-a t)\right)\right] .
$$

This is d'Alembert's well known formula.

\section{References}

[1] M. Miyake, Global and local Goursat problems in the class of holomorphic or partially holomorphic functions, J. Differential Equations 39 (1981), 445-463.

[2] J. Persson, On the global and local non-characteristic Cauchy problems when the solutions are holomorphic functions or analytic functionals in space variables, Ark. Mat. 9 (1971), 171-180.

[3] B. Y.Sternin and V. E. Shatalov, On some integral transformations of complex-analytic functions, Dokl. Akad. Nauk SSSR 280 (1985), 553-556.

[4] L. I. Ronkin, Introduction to the Theory of Entire Functions in Several Variables, Nauka, Moscow, 1971. 